\title{
It's still all about graphene
}

\section{This two-dimensional crystal keeps playing a dominant role in physics and materials science.}

Andre Geim and Kostya Novoselov were awarded the 2010 Nobel Prize in Physics "for groundbreaking experiments regarding the two-dimensional material graphene". The announcement came after only six years from their first publication on this material ${ }^{1}$. During this time, however, interest in this single layer of graphite has risen exponentially, and has resulted in over 5,000 papers so far.

The unique electronic properties have arguably been the most intriguing aspect of graphene since the first experiments were reported. To name just the most obvious, electrons in this material behave like massless Dirac fermions, and have exceptionally high mobility that allows observation of quantum phenomena up to room temperature ${ }^{2,3}$.

Recently, it was demonstrated that local strain can induce effective magnetic fields of up $300 \mathrm{~T}$ in graphene ${ }^{4}$. But it is the combination of such intriguing properties with the high strength, flexibility and potential for chemical modification that has raised huge interest in graphene among condensed-matter physicists and materials scientists.

The announcement of the Nobel Prize is often surrounded by controversy. To cite just some recent cases, many would have considered it appropriate for Stuart Parkin to share the 2007 prize in physics with Albert Fert and Peter Grünberg, for his contribution to the development of applications of giant magnetoresistance. Not to mention the fact that the list of laureates for the development of the CMK matrix (from Cabibbo-Kobayashi-Maskawa) in 2008 did not include Cabibbo. Graphene is no exception: it has been suggested that the background information provided by the Nobel Committee contained several inaccuracies and omissions that resulted in overlooking the work of other scientists ${ }^{5}$.

If errors have been made they must be corrected. And indeed, the field of graphene research has developed also through the important contributions of other scientists such as Philip Kim, Walt de Heer and Rod Ruoff, among others. But this can in no way deny the leading roles that Geim and Novoselov have played. In their 2004 Science paper they showed that, unexpectedly, graphene could exist in its two-dimensional crystallographic form ${ }^{1}$. The simplicity of their exfoliation method made it possible for a wide scientific community to access the material experimentally, which the two scientists have always been keen to encourage,

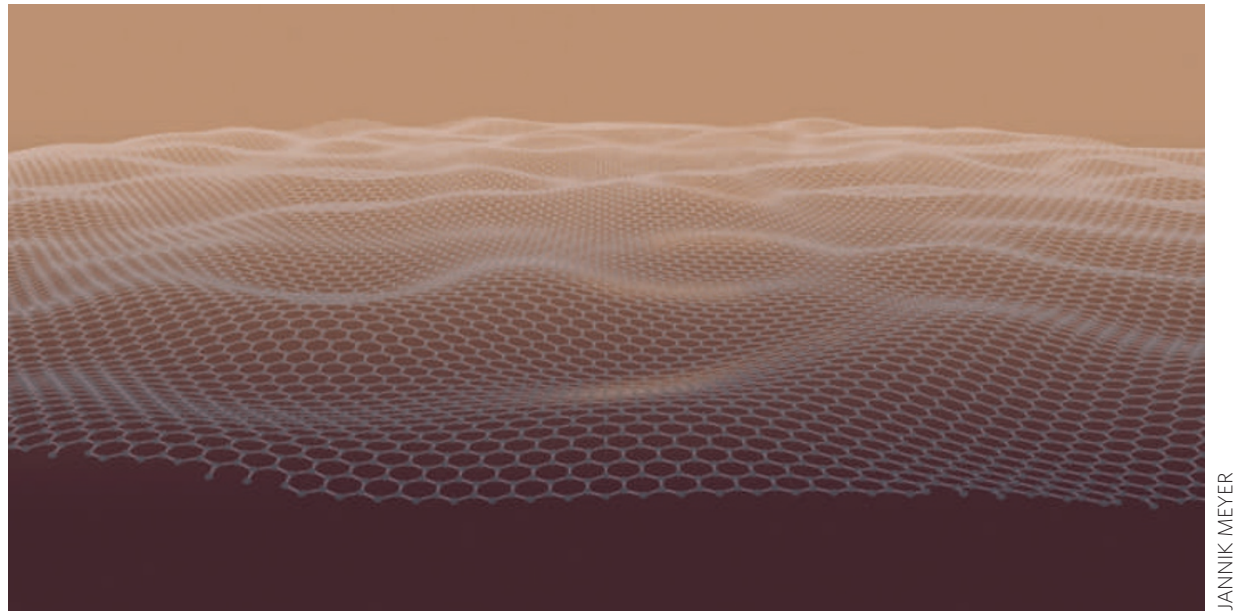

even by openly inviting colleagues to their lab in Manchester to teach them the practical steps needed to obtain and recognize single layers. They have since produced a bulk of work on the fundamental electronic properties but have also started exploring the multiple facets of this material, including optical transmission ${ }^{6}$, the capability of sensing molecules with extreme resolution ${ }^{7}$, and the possibility of creating new two-dimensional molecules - first by hydrogenation of graphene $e^{8}$ and more recently by its fluorination ${ }^{9}$. To quantify their contribution to the field, their publications since 2004 have generated well over 15,000 citations.

Many also felt that the Nobel Prize for graphene came too soon, especially as dreams of graphene-based applications have yet not become reality. There is no doubt that despite the hype, whether the material will revolutionize electronics remains an open question. There are many problems of production costs and integration with existing devices, mainly due to the absence of a semiconducting gap in pristine graphene.

But aside from the fact that applications were not the reason for the award, it would be unfair to deny that promising results have been obtained, even after such a short time. The exfoliation method will never be used on industrial scales. But impressive advances have been made, for example in the production of graphene by chemical vapour deposition ${ }^{10}$. The high electrical conductivity, combined with its strength, flexibility and transparency make graphene an ideal material for applications in flexible optoelectronic devices. It was recently demonstrated that largescale films can be synthesized and used for touch-screen displays ${ }^{11}$, and there are reasons to believe that the first products of this type will reach the market soon. High-frequency transistors are other devices that were predicted to benefit from the exceptionally high electron mobility. Transistors operating at $100 \mathrm{GHz}$ were demonstrated in May $2010^{12}$ and claims of $300 \mathrm{GHz}$ were reported only a few months later ${ }^{13}$. Graphene and graphenebased composites have also shown promising results for photonic devices, for example in laser components and photodetectors ${ }^{14}$. And although they are still at an exploratory stage, there are research efforts in various other aspects of materials science, from the use in composites for mechanical applications to energy-storage-related structures and bio-sensing.

Whether the work initiated by Geim and Novoselov will substantially influence our everyday life remains to be seen. But without a doubt it has already massively influenced the scientific community and it won't stop doing so for a long time.

\section{References}

1. Novoselov, K. S. et al. Science 306, 666-669 (2004).

2. Geim, A. K. \& Novoselov K. S. Nature Mater. 6, 183-191 (2007).

Novoselov K. S. et al. Science 315, 1379 (2007)

4. Levy, N. et al. Science 329, 544-547 (2010).

5ature 468, 486 (2010)

Nair, R. R. et al. Science 320, 1308 (2008).

7. Schedin, F. et al. Nature Mater. 6, 652-655 (2007).

8. Elias, D. C. et al. Science 323, 610-613 (2009).

9. Nair, R. R. et al. Small doi:10.1002/smll.201001555 (2010)

10. Li, X. S. Science 324, 1312-1314 (2009).

11. Bae, S. et al. Nature Nanotech. 5, 574-578 (2010).

12. Lin, Y. M. et al. Science 327, 662 (2010).

13. Liao, L. Nature $467,305-308$ (2010).

14. Bonaccorso, F., Sun, Z., Hasan, T. \& Ferrari, A. C. Nature Photon. 4, 611-622 (2010).

Corrected online: 22 December 2010 


\section{Correction}

In the Editorial 'It's still all about graphene'

(Nature Mater. 10, 1; 2011), the first name of the person the image is credited to was spelt incorrectly as Yannik; it should have read Jannik. Corrected in the HTML and PDF versions after print: 22 December 2010 